\title{
Vaisseaux \\ lymphatiques \\ et cancer
}

> La survie et le développement des tumeurs dépendent d'événements et d'échanges biologiques nutritionnels et respiratoires assurés par le sang et la lymphe. La prolifération tumorale est associée à un accroissement des réseaux vasculaires soit à proximité de la tumeur, soit en intra-tumoral. Les tissus tumoraux sont capables d'augmenter leurs approvisionnements, en fonction de leurs besoins au sein de l'organisme, en orientant et en optimisant le développement des vaisseaux péri-tumoraux. La production par les cellules cancéreuses de facteurs de croissance stimulant la néoformation des vaisseaux lymphatiques constitue une des adaptations responsables de la propagation métastatique. Au cours du développement tumoral, le système lymphatique est considéré dans de nombreux cas de cancers comme la voie primaire de dissémination des métastases. L'étude de la mise en place du système lymphatique et éventuellement de son blocage sont des points importants à considérer en oncologie. <

Le système lymphatique est peu connu alors qu'il joue probablement un rôle de premier plan dans certaines pathologies. II constitue un vaste ensemble de vaisseaux qui drainent une partie des liquides interstitiels sous forme de lymphe vers les organes lymphoïdes et vers le cœur. Leur trajet parcourt, en certaines régions, des ganglions lymphatiques qui sont à la fois des filtres mécaniques et des barrières immunitaires s'opposant à la progression d'éléments pathogènes véhiculés par la lymphe. Parmi ces éléments se trouvent parfois des cellules cancéreuses qui peuvent soit être détruites dans les ganglions, soit s'y développer avant de poursuivre leur propagation vers d'autres organes. Ainsi, le système lymphatique constitue à la fois un élément essentiel du système immunitaire et une voie majeure de dissémination des cellules métastatiques dans de

Article reçu le 28 février 2005, accepté le 22 juillet 2005.

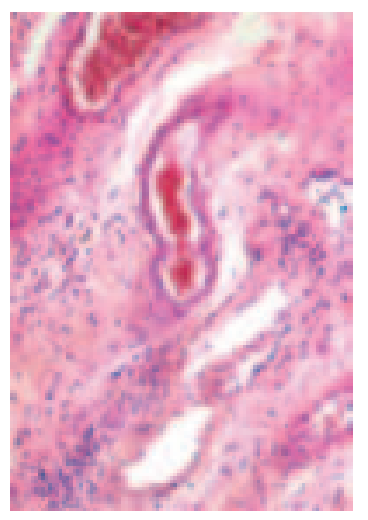

Médecine moléculaire humaine EA 3839, Faculté de Médecine, 2, rue du Docteur Marcland, 87025 Limoges Cedex, France.

nombreux cancers tels que ceux du sein, de dany.leclers@unilim.fr la sphère $\mathrm{ORL}$ ou du testicule $[1,2]$. Étudier son organisation et ses mécanismes de mise en place est donc une étape indispensable avant d'envisager des voies thérapeutiques visant ce système.

\section{Organisation générale et rôles du système lymphatique}

Le système lymphatique est formé de vaisseaux qui drainent les liquides interstitiels de l'organisme vers des organes lymphoïdes comme les ganglions lymphatiques, la rate et les amygdales ainsi que vers les organes les plus évolués tels que le thymus et la moelle osseuse (Figure 1A). Le système vasculaire lymphatique est organisé en un fin réseau de canalicules dans lequel circule de la lymphe. Ce réseau permet de réguler en permanence le niveau d'hydratation tissulaire ainsi que l'évacuation de certains déchets cellulaires. La circulation de la lymphe à l'intérieur des voies lymphatiques est unidirectionnelle, des tissus vers les ganglions lymphatiques puis vers le réseau veineux. Des valvules tronconiques constituent un système anti-retour [3] (Figure 1B). Ces structures sont plus nombreuses que dans les vaisseaux sanguins. Les mouvements liquidiens à l'intérieur du réseau s'effectuent d'une manière similaire à celle de la circulation sanguine veineuse, mais la pression à l'intérieur des voies lymphatiques est plus faible et la circulation y est donc plus lente. Ces mouve- 
ments liquidiens sont en particulier sous la dépendance des compressions liées aux contractions musculaires, aux mouvements respiratoires et, plus faiblement, de l'onde du pouls artériel [4].

Les convergences des voies lymphatiques aboutissent au drainage de la lymphe d'origine tissulaire vers les ganglions lymphatiques avant un retour vers la circulation sanguine. Les canaux thoraciques de la partie inférieure de l'organisme s'anastomosent à la base du thorax pour former le canal thoracique qui rejoint au niveau du médiastin le tronc lymphatique du bras gauche et de l'hemi-tête gauche pour finalement rejoindre la circulation sanguine en se jetant dans les veines jugulaire et sous-clavière gauche au niveau de la base du cou. Le drainage de la lymphe provenant de la partie droite du corps aboutit à la circulation générale par l'intermédiaire de la veine sous-clavière droite. Les ganglions lymphatiques sont différenciés selon leur localisation, superficielle (rétro- et sous-maxillaires, mentonniers, carotidiens, sus-claviculaires, occipitaux, axillaires, épitrochléens, inguinaux, rétro-poplités...) ou profonde (para-trachéaux, inter-trachéo-bronchiques, inter-bronchiques, mésentériques, aorticaux-mésentériques, lombaires, iliaques, pelviens, rétro-cruraux...). On en dénombre entre cinq cents et mille répartis dans tout l'organisme. Les veines lymphatiques, qui sont les plus gros vaisseaux lymphatiques, ont une paroi formée de trois couches : la plus interne fine, transparente, légèrement élastique, est constituée de cellules endothéliales. La couche intermédiaire est formée de cellules musculaires lisses et de fibres élastiques transversales. La couche externe formée de cellules musculaires lisses orientées longitudinalement et transversalement, formant une protec- tion cellulaire contractile et permettant les connexions vasculaires avec les structures voisines. Les capillaires lymphatiques sont, quant à eux, formés d'une couche unique de cellules endothéliales de forme aplatie et leur diamètre est généralement compris entre 20 et 30 $\mu \mathrm{m}$, supérieur à celui des capillaires sanguins (Figure $2 A$ ). Ils sont moins nombreux que ces derniers et leurs parois sont plus souples. Par ailleurs, l'endothélium des capillaires lymphatiques est ponctué de jonctions lâches de type macula adherens, contrairement aux capillaires sanguins largement pourvus de jonctions plus étroites, de type zonula occludens. Le trajet de ce réseau chemine en général parallèlement aux systèmes vasculaires sanguins artériel et veineux. Les capillaires lymphatiques se regroupent par confluences successives pour former des canalicules puis des collecteurs dont on distingue deux types, les collecteurs principaux et terminaux [5] (Figure 2B).

La circulation lymphatique permet la récupération de certaines macromolécules, principalement des protéines, ayant filtré à travers la barrière vasculaire sanguine. Cette capture est réalisée au niveau des fins capillaires lymphatiques. Le système lymphatique est également impliqué dans l'élimination des débris cellulaires, de déchets du métabolisme et de certains antigènes après leur passage au niveau des ganglions lymphatiques. Cette fonction d'épuration est corrélée

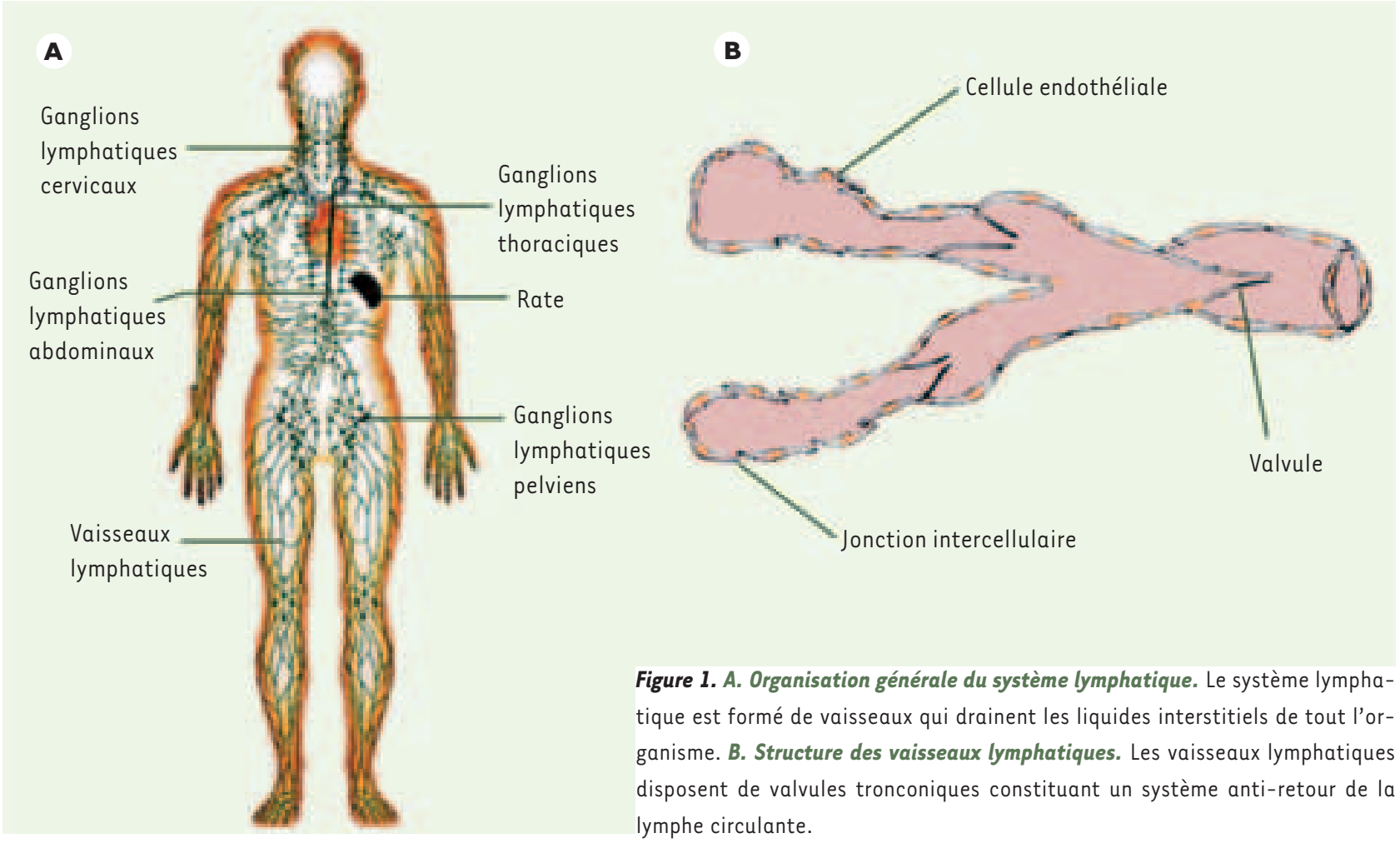


avec la mise en contact des anticorps et des antigènes pour faciliter l'activation des défenses de l'organisme. D'un point de vue immunitaire, les vaisseaux lymphatiques permettent les échanges entre les éléments du système lymphoïde. Ainsi, après le drainage des zones périphériques par le réseau lymphatique, les organes lymphoïdes secondaires, essentiellement la rate, les ganglions lymphatiques, les amygdales et l'appendice, se trouvent être les sites de l'élaboration de la réponse
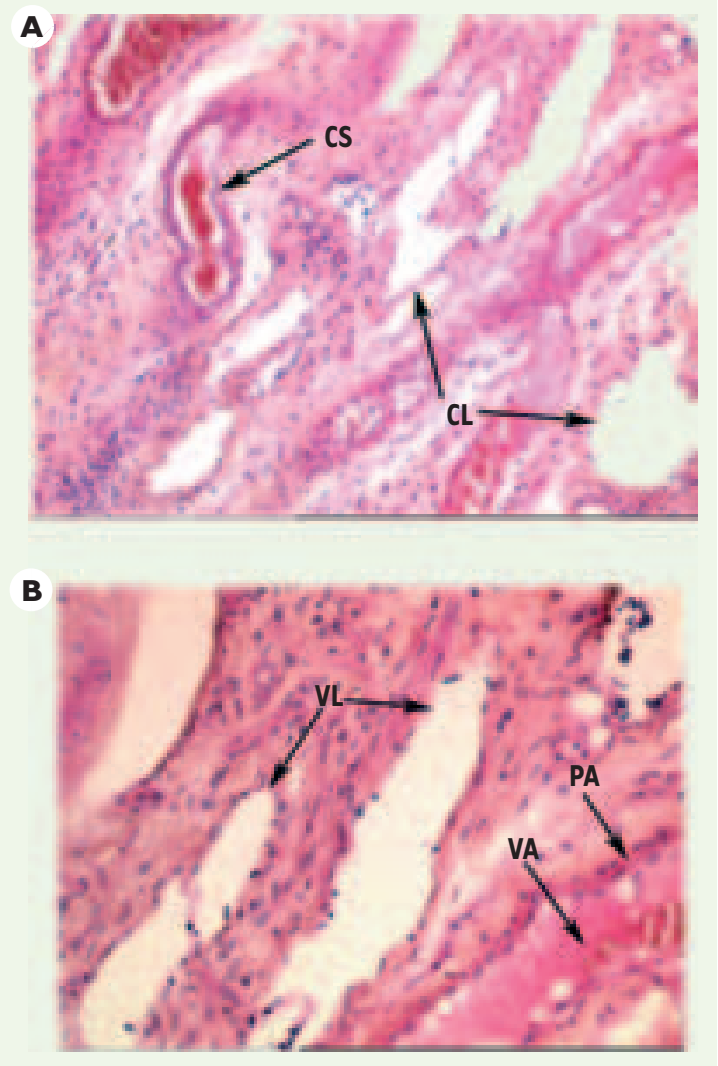

Figure 2. A. Observation microscopique de vaisseaux sanguins et lymphatiques (hématéine-éosine-safran x 100, Pr F. Paraf, Laboratoire d'anatomie pathologique, CHU de Limoges). Les capillaires lymphatiques ont un diamètre généralement plus important que les capillaires sanguins, favorisant la dissémination métastatique dans de nombreux types tumoraux. CS: capillaire sanguin (contenant des hématies); $C L$ : capillaire lymphatique (contenant des lymphocytes). B. Observation microscopique de structures vasculaires (hématéine-éosinesafran $x 200$, Pr F. Paraf, Laboratoire d'anatomie pathologique, CHU de Limoges). Le trajet du réseau lymphatique se situe souvent parallèlement à celui des systèmes vasculaires sanguins artériels et veineux, ce qui favorise les échanges vasculaires. VL: vaisseau lymphatique; VA: vaisseau artériel; PA : paroi artérielle. immunitaire. En effet, à la sortie des organes lymphoïdes primaires, les lymphocytes $B$ et $T$ sont devenus immunocompétents mais ne sont pas encore actifs. Ils migrent alors pour coloniser les organes lymphoïdes secondaires, lieu de la rencontre entre antigènes et lymphocytes immunocompétents. Ces éléments de la réponse immunitaire peuvent également atteindre tous les territoires de l'organisme en circulant librement par les voies sanguines et lymphatiques qui relient entre eux ces organes [6]. Enfin, le système lymphatique assure le transport des lipides au foie après leur absorption au niveau de l'intestin grêle. Par ailleurs, il tient un rôle important dans le maintien de l'homéostasie en contribuant à la constance de l'hydratation tissulaire et en permettant l'évacuation des protéines et d'autres macromolécules des liquides interstitiels (Figure 3).

\section{Ontogenèse du système lymphatique}

\section{Au niveau cellulaire}

La formation du système lymphatique au cours de l'embryogenèse s'effectue après celle du système cardiovasculaire, mais lui est étroitement associée. Le système lymphatique se constitue en partie par la différenciation de cellules lymphangioblastiques du

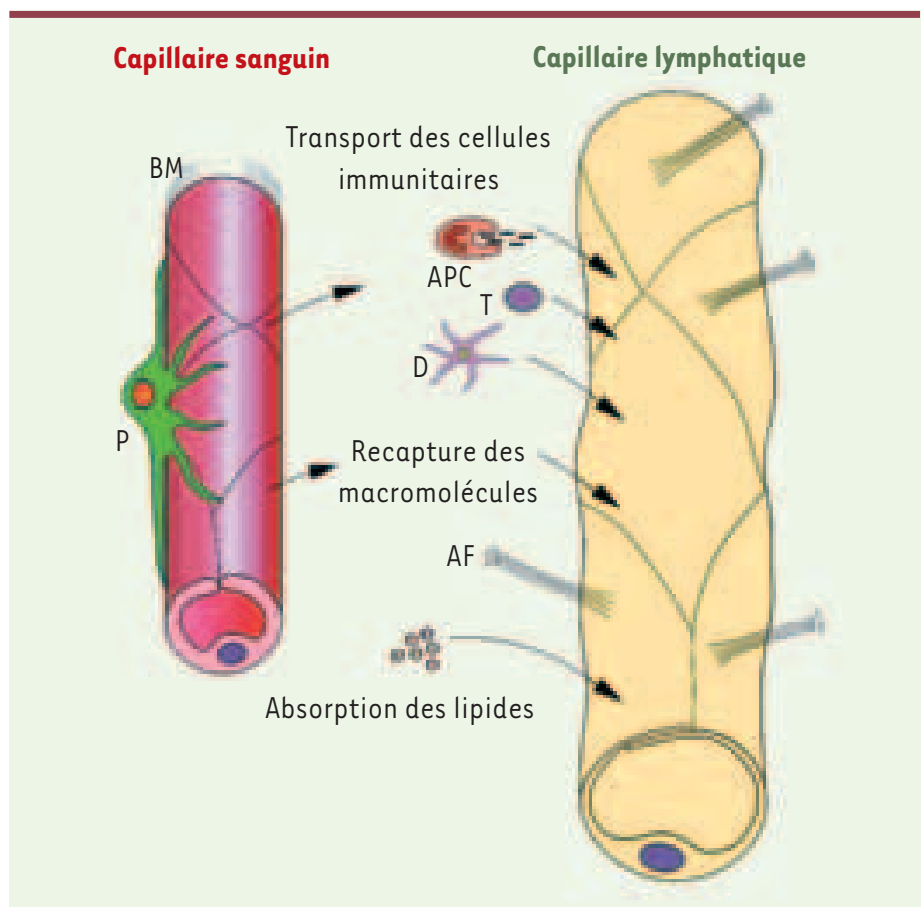

Figure 3. Principales fonctions des capillaires lymphatiques. Outre l'élimination des déchets métaboliques et le maintien de l'homéostasie, les vaisseaux lymphatiques sont impliqués dans la circulation des éléments de la réponse immunitaire, la recapture des macromolécules et le transport des lipides. BM: membrane basale; $P$ : péricyte ; $A P C$ : cellule présentatrice d'antigène ; $T$ : lymphocyte T; D : dendrocyte ; AF : filament d'anchor. 
mésenchyme primaire et par la formation de six sacs lymphatiques fœtaux induits par bourgeonnement à partir de la vascularisation initiale [7]. Les sacs lymphatiques fœtaux se forment à partir de grosses veines centrales préexistantes dans les régions jugulaire et péri-mésonéphrique à la suite de multiples fusions des capillaires régionaux. Chez l'homme, ces sacs primitifs apparaissent entre la sixième et la septième semaine du développement embryonnaire, soit environ quatre semaines après le début de la formation des premiers vaisseaux sanguins [8]. Aucune des connexions veinolymphatiques n'est alors réalisée, à l'exception de celles de la région du canal thoracique gauche. Les premiers vaisseaux apparaissent alors par bourgeonnement à partir de ces sacs primitifs avant leur progression vers les régions périphériques. Par ailleurs, l'origine mésenchymateuse du système lymphatique est confirmée par la présence de lymphangioblastes durant le développement embryonnaire, initialement localisés au niveau du mésoderme paraxial et splanchnopleural à l'origine de la formation d'une partie du système lymphatique [9].

\section{Au niveau moléculaire}

La formation des voies lymphatiques est assujettie à un certain nombre de régulations à l'origine de la transduction du signal lymphangiogénique. Ce processus est initié par l'activation d'un récepteur nommé VEGFR-3 (vascular endothelial growth factor receptor-3) localisé sur la membrane plasmique des cellules endothéliales des vaisseaux lymphatiques. Le récepteur VEGFR-3 est spécifiquement activé par les facteurs de croissance VEGF-C et VEGF-D (vascular endothelial growth factor-C/-D) [10], tandis que d'autres facteurs de croissance (PIGF, VEGF-A, VEGF-B, VEGF-C, VEGF-D et VEGF- $\varepsilon$ ) sont responsables de l'activation des protéines VEGFR-1 et VEGFR-2 induisant l'activation de la voie de transduction du signal angiogénique [11] (Figure 4A). Lorsque deux molécules de VEGF-C ou -D se fixent sur leurs récepteurs VEGFR3, les monomères des récepteurs se dimérisent au sein de la membrane cytoplasmique. Ce processus engendre un rapprochement des domaines à activité tyrosine kinase aboutissant à une transphosphorylation des domaines intra-cytoplasmiques des récepteurs. Les récepteurs VEGFR-3 phosphorylés deviennent alors capables de se lier à une protéine de couplage GRB2 (growth factor receptor-bound protein 2), elle-même susceptible d'interagir avec une molécule messagère SOS (son of sevenless). SOS se lie au complexe RAS-GDP inactif accolé à la membrane plasmique. Le remplacement de GDP (guanosine 5'-diphosphate) par GTP (guanosine 5'-triphosphate) permet la transformation en forme active RAF, amorçant une cascade de protéine kinases MEK (MAP/ERK kinase). Ce processus aboutit à l'activation d'une protéine ERK (extracellular signal regulated kinase) à l'origine de l'activation des facteurs de transcription nucléaires des gènes de la lymphangiogenèse (Figure $4 B$ ). Il a été décrit récemment qu'une activation de la transduction du signal lymphangiogénique pouvait éventuellement survenir à la suite de l'interaction de VEGF-A avec le récepteur VEGFR-2 [12] mais cette dernière est actuellement discutée.

\section{Marqueurs spécifiques de la lymphangiogenèse}

Pour détecter et quantifier la néovascularisation lymphatique, il est indispensable de disposer de molécules spécifiques des vaisseaux lymphatiques. Aucune molécule ne constitue, à l'heure actuelle, un marqueur garantissant une fiabilité rigoureuse pour révéler la présence des cellules de l'endothélium des vaisseaux lymphatiques. Cette particularité constitue l'un des écueils principaux limitant les investigations concernant le remodelage lymphatique en pathologie humaine. Les récepteurs VEGFR-3, s'ils sont systématiquement mis en évidence au niveau des voies lymphatiques, sont également exprimés et détectés dans d'autres tissus ou organes tels que le cœur, les poumons ou les reins [13]. Ils se trouvent être, par ailleurs, surexprimés dans de très nombreux organes au cours de l'embryogenèse. Le récepteur VEGFR-3 n'est donc pas un marqueur idéal de la lymphangiogenèse, d'une manière comparable à d'autres molécules comme LyVE-1 (lymphatic vessel endothelial hyaluronan receptor-1) et la podoplanine qui sont également exprimés au niveau des vaisseaux lymphatiques [14].

$\varepsilon$ n effet, LyVદ-1 est un récepteur d'un glycosaminoglycane, l'acide hyaluronique, constituant de la matrice extracellulaire dont le transport s'effectue par les vaisseaux lymphatiques. Il est détecté sur la face externe de l'endothélium des vaisseaux lymphatiques de nombreux tissus $[15,16]$. Cependant, il est désormais avéré que son expression n'est pas restreinte aux seuls vaisseaux lymphatiques. LyVE-1 est détecté en particulier au niveau des cellules de Kupffer, macrophages localisés sur la face luminale des sinusoïdes hépatiques [17].

La podoplanine est une glycoprotéine de la membrane des podocytes située à proximité des récepteurs VEGFR3, au niveau des petits vaisseaux lymphatiques. II a été démontré que cette molécule peut être utilisée en tant que marqueur de l'endothélium des capillaires lymphatiques [18]. Sa fiabilité est cependant discutée.

II existe un autre marqueur des néo-vaisseaux lymphatiques: il s'agit de la protéine Prox 1 (prosperorelated homeobox 1) qui joue un rôle fondamental dans le développement précoce du système nerveux central. Elle pourrait réguler l'expression génique et le développement des neurones natifs post-mitotiques indifférenciés. Prox 1 est considérée comme un marqueur approximatif de la lymphangiogenèse [19] car son expression a notamment été retrouvée au niveau cardiaque, hépatique et cérébral [20-22].

La spécificité relative de ces marqueurs limite à ce jour leur utilisation essentiellement à des fins d'outils d'évaluation dévolus notamment à la quantification d'éventuelles inhibitions de la lymphangiogenèse péri-tumorale 
dans la mise au point de nouvelles thérapeutiques anticancéreuses. Cependant, l'utilisation simultanée de plusieurs de ces marqueurs permet d'améliorer la certitude quant à la détection des vaisseaux lymphatiques. Ainsi, certaines études récentes ont défini le taux d'expression des marqueurs VEGR-3 et podoplanine comme un élément fortement prédictif de l'atteinte ganglionnaire pour certains cancers [23].

\section{Système lymphatique et pathologies}

\section{Lymphœdèmes}

Les pathologies les plus fréquentes du système lymphatique se traduisent par des lymphœdèmes. Ces derniers correspondent à l'accumulation de liquide lymphatique dans les espaces interstitiels, particulièrement au niveau de la graisse sous-cutanée et concernent généralement les membres. Les lymphœdèmes sont dus soit à un blocage mécanique ou post-radiothérapie de l'évacuation de la lymphe, soit à une augmentation ponctuelle de la quantité de lymphe à évacuer. II en résulte une accumulation anormale de protéines tissulaires avec œdème, inflammation chronique et fibrose. On distingue deux types de lymphœdèmes. Les lymphœdèmes dits primaires sont dus à des anomalies morphologiques du système lymphatique. Ils sont généralement d'origine familiale ou héréditaire (maladie de Milroy) avec une forte prédominance chez les femmes. Certains apparaissent à la puberté (maladie de Meige) ou à la suite d'une grossesse.
Les lymphœdèmes secondaires sont associés, quant à eux, à une destruction du système lymphatique. Ils peuvent être déclenchés par une intervention chirurgicale (curage ganglionnaire), un traumatisme, un envahissement néoplasique, une infection (lymphangite), une parasitose (filariose) ou un traitement par radiothérapie.
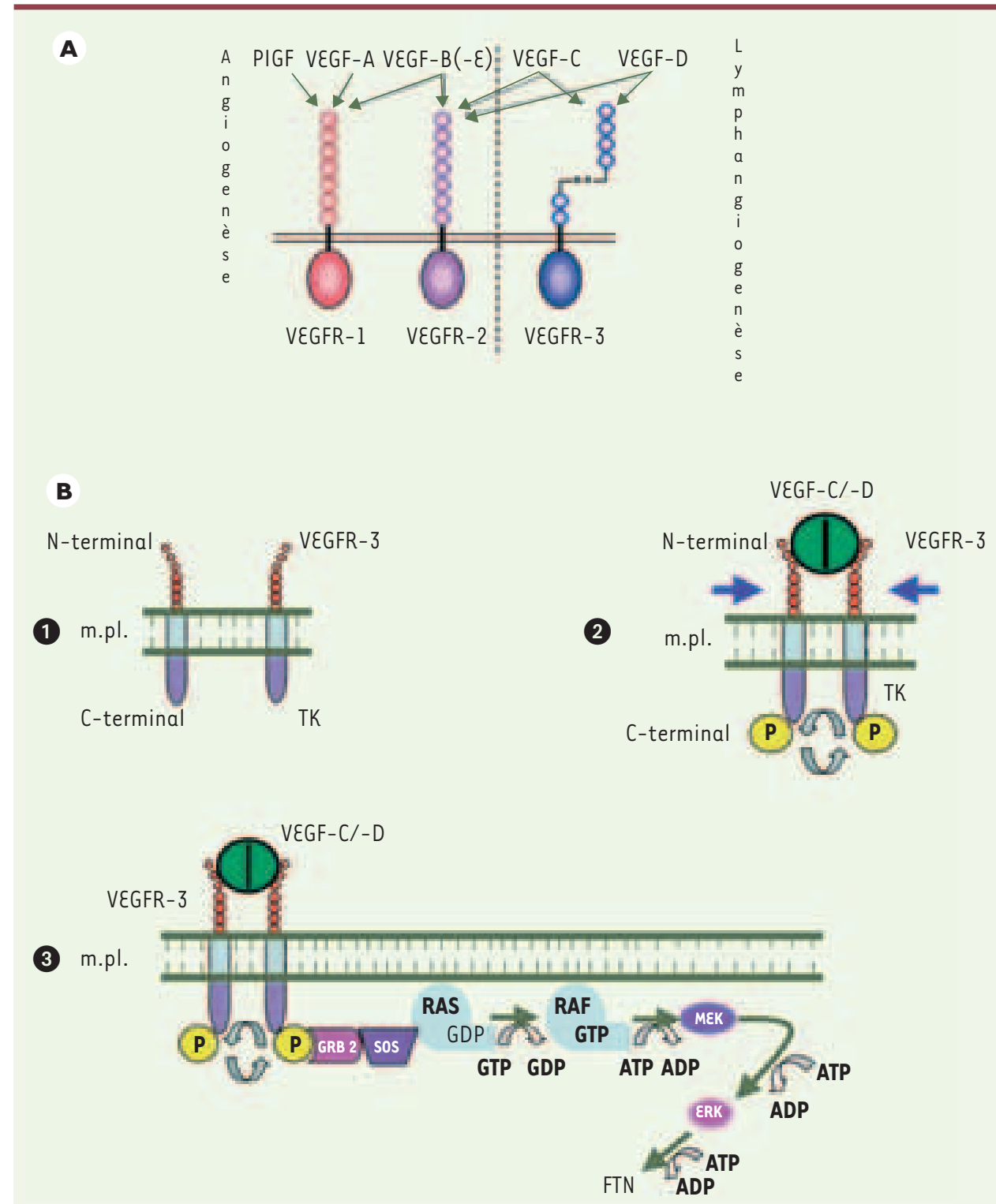

Figure 4. A. Facteurs de croissance et récepteurs des synthèses vasculaires. La synthèse des néo-vaisseaux est sous la dépendance de trois récepteurs de facteurs de croissance : VEGFR-1 et VEGFR-2 pour la synthèse de nouveaux vaisseaux sanguins, VEGFR-3 pour la synthèse de nouveaux vaisseaux lymphatiques. Ces récepteurs sont activés (flèches vertes) par différents facteurs de croissance (voir texte). PIGF : placental growth factor ; VEGF : vascular endothelial growth factor. B. Mécanisme d'activation de la lymphangiogenèse. La transphosphorylation de deux monomères de récepteur VEGFR-3 (1, 2) permet l'activation d'une cascade de kinases (3) impliquant les protéines RAS, RAF, MEK (MAP/ERK kinase) et ERK (extracellular signal regulated kinase) (voir texte). GRB2 : growth factor receptor-bound protein 2 ; SOS : son of sevenless ; FTN : facteur de transcription nucléaire; m.pl. : membrane plasmique. 


\section{Lymphangiomes et lymphangiosarcomes}

Les lymphangiomes sont des lésions prolifératives bénignes et rares caractérisées par une hypervascularisation lymphatique et qui aboutissent à la constitution d'une dilatation molle, translucide, dépressible, aux parois minces sur laquelle se forment des amas de vésicules. Les lymphangiomes sont généralement observés dans le creux axillaire, sur la poitrine, dans la bouche et sur la langue. Leur évolution est chronique, émaillée de poussées douloureuses très fébriles, phénomène que l'on retrouve dans le lymphœdème. Ils s'associent fréquemment à une prolifération des vaisseaux sanguins (hémolymphangiome). Certains lymphangiomes sont acquis, en particulier à la suite d'une radiothérapie. Les lymphangiomes kystiques développés dans la racine du mésentère peuvent engendrer des masses de taille variable, parfois très volumineuses, respectant le parenchyme et se moulant sur les structures voisines. Classiquement, en échographie, il s'agit d'une masse molle, cloisonnée avec des cavités kystiques de taille variable. Le diagnostic différentiel peut se poser avec celui d'un lymphome, d'une duplication digestive ou d'un kyste volumineux d'origine ovarienne quand il présente un développement abdominal préférentiel.

Le lymphangiosarcome ou syndrome de Stewart-Treves constitue une complication rare, tardive et souvent méconnue des lymphœdèmes chroniques post-thérapeutiques. Son étiologie a été secondairement élargie aux lymphœdèmes chroniques de toutes origines. II s'agit d'une tumeur maligne développée à partir des cellules de l'endothélium de vaisseaux lymphatiques. Les lymphangiosarcomes surviennent la plupart du temps au niveau du membre supérieur à la suite d'une mastectomie totale, mais ils viennent également parfois compliquer les lymphœdèmes idiopathiques. Le diagnostic doit être évoqué devant l'apparition sur un lymphœdème chronique ancien d'une tuméfaction violacée d'allure vasculaire, souvent multinodulaire. Ces petits nodules insérés dans le derme peuvent s'ulcérer ou se fusionner plus ou moins les uns aux autres pour former des plaques étendues. Au stade localisé, le traitement repose surtout sur la chirurgie radicale. La chimiothérapie proposée implique habituellement les anthracyclines, dont l'utilisation est difficile chez les personnes âgées du fait de leur toxicité.

\section{Système lymphatique et processus tumoral}

\section{Échanges avec les cellules tumorales}

Le réseau des vaisseaux lymphatiques intervient dans les échanges entre les cellules tumorales et le milieu environnant en constituant une interface entre les cellules et le sang. En effet, l'approvisionnement des cellules en nutriments, qu'elles soient tumorales ou non, ne se fait pas directement par le sang: du plasma s'infiltre continuellement entre les cellules, constituant la lymphe interstitielle qui baigne les tissus et assure le transport intermédiaire des nutriments jusqu'aux cellules. Ces échanges sont bidirectionnels et permettent également l'évacuation des déchets du métabolisme cellulaire. Cette détoxication du milieu péri-tumoral est indispensable à la croissance optimale de ces tissus dont le métabolisme prolifératif est fortement producteur de résidus dénaturés, finalement canalisés dans les voies lymphatiques avant leur élimination via la circulation sanguine [24].
Voies lymphatiques et dissémination métastatique Les cellules cancéreuses traversant un ganglion lymphatique y sont généralement séquestrées. Elles sont alors détruites mais peuvent, dans certaines circonstances, s'y développer, formant ainsi un foyer tumoral secondaire. C'est souvent le cas dans les cancers du sein où le ganglion sous-axillaire constitue généralement le premier relais métastatique. Dans de tels types de cancer, les vaisseaux lymphatiques peuvent être altérés lorsque la tumeur exerce une compression gênant la circulation lymphatique. On peut alors déplorer l'apparition d'un lymphœdème autrefois souvent à l'origine d'un gros bras. C'est également le cas dans le traitement des cancers du sein avec curage ganglionnaire impliquant l'ablation des ganglions et des vaisseaux lymphatiques locaux. Il existe, par ailleurs, des tumeurs spécifiques du système lymphatique appelés lymphomes. Ils peuvent se développer à partir des lymphocytes B ou T. Dans ces pathologies, le dépistage se fait principalement par palpation des ganglions superficiels au niveau du cou, des aisselles et de l'aine. Les ganglions profonds sont explorés par radiographie: radiographie pulmonaire pour ceux du médiastin dans le thorax, scanographie ou lymphographie pour ceux de l'abdomen ou par la tomographie par émission de positons (PET-scan).

Le premier ganglion concerné par un éventuel envahissement axillaire issu d'une tumeur primitive est appelé le ganglion «sentinelle». L'étude histologique après biopsie de ce ganglion, en cas de doute, est donc théoriquement prédictive de celle de l'intégralité de la chaîne ganglionnaire. Des techniques de marquage par coloration ou radio-isotope du ganglion sentinelle destinées à le localiser puis à l'exciser pour examen anatomopathologique ont été développées afin de préciser certains diagnostics. Le marqueur est capté puis drainé par les capillaires lymphatiques jusqu'au ganglion sentinelle qui est alors repéré, prélevé et analysé [25].

Au cours du développement tumoral, on constate généralement une augmentation de la vascularisation péri-tumorale. Au niveau des voies lymphatiques, il est couramment observé un accroissement significatif de la longueur et du diamètre des vaisseaux ainsi que l'apparition de nouvelles ramifications vasculaires. Cependant, les tumeurs ne possèdent pas de système vasculaire lymphatique intrinsèque, mais des vaisseaux dilatés dans le stroma péri-tumoral, pénétrant occasionnellement en périphérie dans la tumeur. Par ailleurs, il est désormais connu que les vaisseaux lymphatiques participent à la propagation tumorale en constituant une voie privilégiée de la dissémination métastasique [26]. L'augmentation du nombre et du diamètre des ramifications lymphatiques à proximité des tumeurs 
correspond alors à une forme de potentialisation de la dissémination des métastases. La pénétration des cellules cancéreuses, des espaces péri-ductaux à travers les parois des vaisseaux lymphatiques, s'effectue par diffusion au travers des béances offertes par les sinus lymphatiques ou par digestion protéolytique de la fine matrice des vaisseaux. Les cellules cancéreuses sont alors véhiculées par le flux liquidien, généralement jusqu'à leur entrée dans des ganglions lymphatiques, avant de rejoindre la circulation sanguine par l'intermédiaire du canal thoracique. Elles peuvent également former des micro-emboles obstruant les capillaires lymphatiques, rejoindre alors les tissus à proximité par dégradation protéolytique de la matrice vasculaire et coloniser de nouveaux territoires de l'organisme en formant des foyers tumoraux secondaires.

La dissémination des métastases par les voies lymphatiques représente la voie la plus commune de dissémination des carcinomes, c'est-à-dire des tumeurs cancéreuses qui affectent soit un tissu de revêtement comme l'épiderme ou les muqueuses, soit un tissu glandulaire. Elle est moins fréquente, bien qu'existante, dans le cas des sarcomes, des tumeurs malignes des tissus conjonctifs.

Certains cancers ont un mode de dissémination métastatique qui s'effectue préférentiellement par l'intermédiaire des vaisseaux lymphatiques. Cette disposition est notamment favorisée par la présence de vaisseaux lymphatiques à proximité de la tumeur et par la structure des vaisseaux lymphatiques. Quelques-unes des caractéristiques du réseau lymphatique et des capillaires qui le composent permettent en particulier d'expliquer la promptitude des cellules métastatiques à rejoindre plus facilement le flux lymphatique que le flux sanguin.

Le premier paramètre est l'immense étendue du réseau lymphatique qui irrigue absolument tous les territoires tissulaires, à la seule exception du cerveau, selon des ramifications qui investissent les moindres méandres de l'organisme.

Le second élément réside dans la structure même des capillaires lymphatiques. Ces micro-vaisseaux possèdent un endothélium extrêmement fin formé d'une couche unique de cellules aplaties bordée par une membrane basale interrompue ou inexistante. Leur paroi est plus souple que celle des capillaires sanguins. La dégradation de cet endothélium par les enzymes protéolytiques synthétisées par les cellules métastatiques est donc d'autant plus efficace. De plus, les capillaires lymphatiques présentent un diamètre plus important que les capillaires sanguins et offrent ainsi une surface de contact supérieure pour l'intrusion des cellules malignes. Par ailleurs, l'endothélium lymphatique est ponctué de jonctions intercellulaires lâches de type maculae adherens destinées à faciliter la capture de certaines macromolécules circulantes et qui constituent des portes d'entrées majeures pour les cellules métastatiques. Le flux liquidien à l'intérieur des vaisseaux lymphatiques est aussi significativement plus faible que celui du système sanguin et la différence de pression qui en résulte pourrait favoriser, d'une part, la pénétrabilité à l'intérieur du réseau lymphatique et, d'autre part, la potentialité de formation des micro-emboles souvent à l'origine de foyers secondaires. Les cancers dont la dissémination métastatique est fortement associée au système lymphatique sont, en particulier, les cancers du sein, de la sphère ORL, du poumon, du testicule, de la peau, ainsi que des tumeurs gastriques.

II n'existe pas, à ce jour, d'argument indiscutable permettant de discerner les raisons pour lesquelles certains types tumoraux utilisent la voie lymphatique plutôt que la voie hématogène pour la dissémination des cellules métastatiques. Cependant, on peut envisager qu'un type tumoral exprimant fortement les facteurs de croissance VEGF-C ou VEGF-D favorisera davantage la croissance proximale de néo-vaisseaux lymphatiques, alors qu'un type tumoral exprimant très fortement VEGF-A ou VEGF-B contribuera davantage à l'enrichissement d'une vascularisation sanguine. Ainsi, le type tumoral pourrait déterminer en partie la nature du réseau vasculaire néo-synthétisé à proximité directe de la tumeur, en fonction des facteurs de croissance qu'il synthétise majoritairement. On peut émettre l'hypothèse selon laquelle ces modifications discriminantes de l'environnement péri-tumoral pourraient être déterminantes et augmenter significativement la probabilité de rencontre entre les cellules métastatiques s'échappant du foyer initial et le système vasculaire requis pour véhiculer ces cellules, désignant dans ce cas les facteurs de croissance de l'endothélium des vaisseaux comme l'un des facteurs moléculaires essentiels orientant vers une dissémination lymphatique ou hématogène.

Au cours de la dissémination des cellules métastatiques, un certain nombre de facteurs physiologiques interviennent pour favoriser la migration, l'invasion et la colonisation au niveau de localisations secondaires. Les données susceptibles de distinguer ces événements dans le cas d'une dissémination lymphatique ou hématogène sont aujourd'hui insuffisantes ou indéterminées. Récemment, les chimiokines ont été décrites comme des molécules capables d'activer fortement certaines signalisations intracellulaires telles que l'activation de la voie des MAP-kinases, l'induction de pseudopodes, l'adhérence aux cellules endothéliales, mais également la mobilité cellulaire, la migration et l'invasion tissulaire. Il est désormais prouvé que les cellules tumorales interagissent avec des chimiokines spécifiques de certains tissus, ce qui permettrait d'expliquer en partie la migration et l'invasion orientée des métastases. Par exemple, les cellules de cancer du sein expriment fortement le récepteur CXCR4 qui se lie à la chimiokine CXCL12. Or, la chimiokine CXCL12 est synthétisée spécifiquement par les tissus pulmonaire, osseux, ganglionnaire et hépatique qui se trouvent être les sites de prédilection des métastases du cancer du sein. En revanche, le tissu cutané, qui n'est pas un site métastatique de ce type tumoral, exprime une autre chimiokine, nommée CCL27 qui n'est pas reconnue par le récepteur CXCR4 [27]. 


\section{Perspectives thérapeutiques en cancérologie : inhiber la dissémination des métastases et la progression tumorale}

Une stratégie thérapeutique consistant à limiter au maximum la néovascularisation lymphatique péri-tumorale pourrait constituer un élément substantiel pour limiter la progression et la dissémination tumorale dans certains types de cancers comme les cancers du sein, de la sphère ORL, du testicule, du poumon, de la peau, de la thyroïde ou encore du côlon. Une telle orientation n'est aujourd'hui qu'à l'état de concept, contrairement aux stratégies anti-angiogéniques qui sont fréquemment au centre de l'intérêt des chercheurs.

Les anti-angiogéniques forment désormais une nouvelle classe médicamenteuse non seulement adjuvante, mais qui commence également à être proposée en monothérapie. Par exemple, l'Avastin ${ }^{\circledR}$ (bevacizumab) est un anticorps monoclonal anti-VEGF-A de 149 kDa, conçu pour se fixer au VEGF-A humain et engendrer ainsi une inactivation de la voie de transduction du signal angiogénique sous la dépendance de ce facteur de croissance spécifiquement impliqué dans la croissance et le développement des cellules de l'endothélium des vaisseaux sanguins. Il en résulte une inhibition considérable de la synthèse de nouveaux vaisseaux sanguins péri-tumoraux pouvant ralentir significativement la progression de plusieurs types de cancers. Une étude multicentrique internationale a démontré que l'Avastin ${ }^{\circledast}$ associé à une chimiothérapie par irinotécan, 5-fluoro-uracile et leucovorine (IFL) entraîne une amélioration de la survie des patients atteints de cancers colorectaux métastasés. Sur l'analyse des cas de 813 patients, IFL-placebo versus IFL-Avastin ${ }^{\circledR}$, la durée médiane de survie était de 15,6 mois pour le premier cas et de 20,3 mois pour le second, ce qui correspond à une diminution du risque de mortalité de $34 \%$ avec l'Avastin ${ }^{\oplus}(p<0,001)$. Par ailleurs, des essais cliniques de phase III sont actuellement en cours, utilisant cet agent bloquant de l'angiogenèse tumorale comme adjuvant dans les cancers du rein, du poumon, du sein, de l'ovaire et du pancréas [28].

De telles stratégies de blocage peuvent être envisagées avec d'autres processus physiologiques dont le fonctionnement est directement sous la dépendance de récepteurs membranaires, comme c'est le cas pour la lymphangiogenèse tumorale (VEGFR-3). La densité vasculaire lymphatique à proximité de la tumeur pourrait ainsi être réduite, inhibant la croissance tumorale, d'une part, et réduisant, d'autre part, la probabilité de migration des cellules cancéreuses par les vaisseaux lymphatiques. Par ailleurs, l'hypothèse d'un couplage des stratégies anti-angiogénique et anti-lymphangiogénique est désormais à l'étude et permettrait un ciblage d'autant plus efficace de l'inhibition vasculaire péri-tumorale.

\section{Conclusions}

La lymphangiogenèse tumorale est un processus important dans la biologie et le développement de la plupart des tissus cancéreux. En effet, si les tumeurs bénéficient des structures vasculaires pré-existantes permettant les premières phases de leur développement, le métabolisme prolifératif spécifique de ces cellules requiert la forma- tion de nombreux vaisseaux supplémentaires destinés à subvenir aux besoins très importants de ces structures anormales. Ces synthèses sont en partie contrôlées par les tumeurs elles-mêmes. La néo-vascularisation péritumorale apparaît donc comme un phénomène adaptatif: elle mobilise des éléments de notre organisme en faveur du développement et de la dissémination des tumeurs qui miment les signaux naturels du système vasculaire. En outre, les clés moléculaires de cette messagerie étant désormais connues, il devient envisageable d'utiliser les outils récents de la biologie moléculaire dans de nouvelles stratégies thérapeutiques. $\diamond$

\section{SUMMARY}

\section{Lymphatic vessels and cancer}

Survival and development of tumors depends on nutritional and respiratory biological events and exchanges ensured by blood and lymph. Tumor proliferation is associated with an increase in the vascular networks either near the tumor or intra-tumorally. Tumor tissues are able to increase their provisionment according to their needs while directing and optimizing the development of peritumoral vessels. The production of growth factors stimulating neo-formation of lymphatic vessels by cancer cells constitutes one of the adaptations responsible for metastatic propagation. During tumor development the lymphatic system is considered in many cases of cancer as the primary means of metastasis dissemination. The study of the lymphatic system setting and ways to block it are important points to consider in oncology. $\diamond$

\section{REMERCIEMENTS}

Les travaux de recherche sur la lymphangiogenèse tumorale réalisés au Laboratoire de Biochimie médicale de la Faculté de Médecine de Limoges sont financés par le Comité de la Creuse de la Ligue contre le Cancer.

\section{RÉFÉRENCES}

1. Cunnick GH, Jiang WG, Gomez KF, Mansel RE. Lymphangiogenesis and breast cancer metastasis. Histol Histopathol $2002 ; 17: 863-70$.

2. Stacker SA, Achen MG, Jussila L, et al. Lymphangiogenesis and cancer metastasis. Nat Rev Cancer $2002 ; 2: 573-83$.

3. Swartz MA. The physiology of the lymphatic system. Adv Drug Deliv Rev $2001 ; 50: 3-20$.

4. Adair TH, Vance GA, Montani JP, Guyton AC. Effect of skin concavity on subcutaneous tissue fluid pressure. Am J Physiol 1991 ; 261: H349-53.

5. Jeltsch M, Tammela T, Alitalo K, Wilting J. Genesis and pathogenesis of lymphatic vessels. Cell Tissue Res 2003 ; 314 : 69-84.

6. Zarzaur BL, Kudsk KA. The mucosa-associated lymphoid tissue structure, function and derangements. Shock 2001 ; 15 : 411-20.

7. Wilting J, Neeff H, Christ B. Embryonic lymphangiogenesis. Cell Tissue Res $1999 ; 297: 1-11$.

8. Van der Putte SC. The development of the lymphatic system in man. Adv Anat Embryol Cell Biol $1975 ; 51$ : 3-60.

9. Wilting J, Papoutsi M, Othman-Hassan K, et al. Development of the avian lymphatic system. Microsc Res Tech 2001 ; 55 : 81-91. 
10. Aprelikova 0, Pajusola K, Partanen J, et al. FLT-4, a novel class III receptor tyrosine kinase in chromosome $5 q 33$-qter. Cancer Res $1992 ; 52: 746-8$.

11. Petrova TV, Makinen T, Alitalo K. Signaling via vascular endothelial growth factor receptors. Exp Cell Res $1999 ; 253: 117-30$.

12. Hong $Y K$, Lange-Asschenfeldt $B$, Velasco $P$, et al. VEGF-A promotes tissue repairassociated lymphatic vessel formation via VEGFR-2 and the alphalbetal and alpha2betal integrins. FASEB J $2004 ; 18$ : 1111-3.

13. Aprelikova 0, Pajusola K, Partanen J, et al. FLT-4, a novel class III receptor tyrosine kinase in chromosome $5 q 33$-qter. Cancer Res $1992 ; 52: 746-8$.

14. Nisato RE, Harrison JA, Buser R, et al. Generation and characterization of telomerase-transfected human lymphatic endothelial cells with an extended life span. Am J Pathol 2004 ; 165 : 11-24.

15. Banerji S, Ni J, Wang SX, et al. LYVE-1, a new homologue of the CD44 glycoprotein, is a lymph-specific receptor for hyaluronan. J Cell Biol 1999 ; $144: 789-801$.

16. Prevo R, Banerji S, Ferguson DJ, et al. Mouse LyVE- 1 is an endocytic receptor for hyaluronan in lymphatic endothelium. J Biol Chem $2001 ; 276: 19420-30$.

17. Mouta Carreira C, Nasser SM, di Tomaso $\varepsilon$, et al. LYVE-1 is not restricted to the lymph vessels: expression in normal liver blood sinusoids and down regulation in human liver cancer and cirrhosis. Cancer Res 2001 ; 61 : 8079-84.

18. Breiteneder-Geleff S, Soleiman A, Kowalski H, et al. Angiosarcomas express mixed endothelial phenotypes of blood and lymphatic capillaries: podoplanin as a specific marker for lymphatic endothelium. Am J Pathol 1999; 154 : 385-94.

19. Hong YK, Foreman K, Shin JW, et al. Lymphatic reprogramming of blood vascular endothelium by Kaposi sarcoma-associated herpes virus. Nat Genet 2004 ; 36 : 683-5.

20. Partanen TA, Paavonen K. Lymphatic versus blood vascular endothelial growth factors and receptors in humans. Microsc Res Tech 2001 ; 55 : 108-21.
21. Zinovieva RD, Duncan MK, Johnson TR, et al. Structure and chromosomal localization of the human homeobox gene Proxl. Genomics 1996 ; 35 : 517-22.

22. Oliver $G$, Sosa-Pineda B, Geisendorf $S$, et al. Prox 1 , a prospero-related homeobox gene expressed during mouse development. Mech Dev $1993 ; 44: 3-16$.

23. Kitadai $Y$, Kodama $M$, Cho $S$, et al. Quantitative analysis of lymphangiogenic markers for predicting metastasis of human gastric carcinoma to lymph nodes. Int J Cancer $2005 ; 115$ : 388-92.

24. Saharinen P, Tammela T, Karkkainen MJ, Alitalo K. Lymphatic vasculature: development, molecular regulation and role in tumor metastasis and inflammation. Trends Immunol $2004 ; 25$ : 387-95.

25. Braat AE, Oosterhuis JW, Moll FC, de Vries JE. Successful sentinel node identification in colon carcinoma using Patent Blue V. Eur J Surg Oncol 2004 ; 30 : $633-7$.

26. Pepper MS, Tille JC, Nisato R, Skobe M. Lymphangiogenesis and tumor metastasis. Cell Tissue Res $2003 ; 314: 167-77$

27. Smith MC, Luker KE, Garbow JR, et al. CXCR4 regulates growth of both primary and etastatic breast cancer. Cancer Res 2004 ; 64 : 8604-12.

28. Hurwitz H, Fehrenbacher L, Novotny W, et al. Bevacizumab plus irinotecan, fluorouracil and leucovorin for metastatic colorectal cancer. N Engl J Med 2004 ; $350: 2335-42$.

TIRÉS À PART

D. Leclers

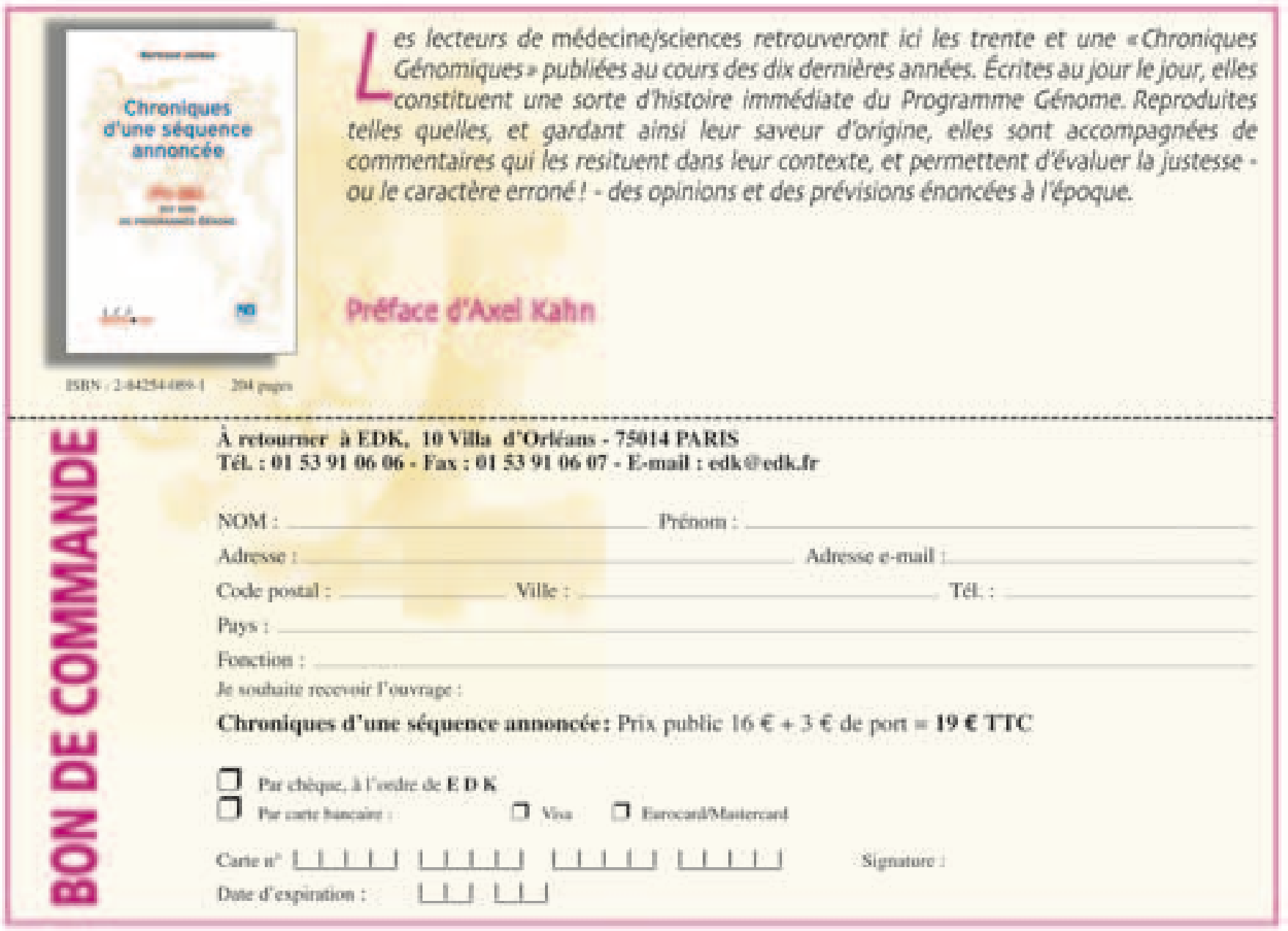




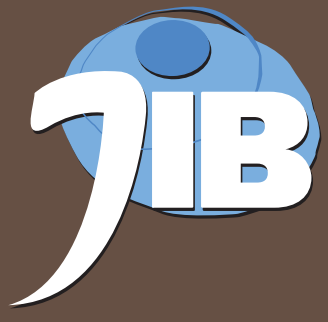

\section{Doupnées \\ Novembre Internationales de Biologie

\section{Programme des conférences scientifiques}

\section{Retrouvez ces informations sur : www.jib-sdbio.fr}

Comité d'Interface INSERM - Biologie Clinique

Mercredi 2 novembre

Thème : Le stress oxydant et ses marqueurs

Modérateur : J. Delattre (Paris)

10h00 : Bases du stress oxydant - Aspects physiologiques

R. Salvayre, Toulouse

10h45: Stress oxydant et expression des gènes. R. Barouki, Paris

11h15: Intérêt des marqueurs du stress oxydant en pathologie et approche thématique - Diabète sucré. P. Gillery, Reims

- Syndrome métabolique. A. Kontush, Paris

- Maladies neurodégénératives. D. Bonnefont-Rousselot, Paris

$12 \mathrm{~h} 15$ Discussion

Modérateur : A. Legrand (Paris)

14h15: Cibles lipidiques et Marqueurs. P. Therond, Paris

15h00: Oxydation des acides aminés et des protéines :

marqueurs plasmatiques et tissulaires. $B$. Friguet, Paris

15h45: Oxydation de l'ADN

- Mécanismes. J. Cadet, Grenoble

- Marqueurs biologiques. A. Favier, Grenoble

16h30: Table Ronde : Quels marqueurs ? Pour quelle indication?

Journée scientifique de la SFBC

Jeudi 3 novembre

Thème : Actualités en biologie clinique

Modérateurs : R. Garnotel (Reims), Y. Piémont (Strasbourg)

09h45: Accueil des participants

10 h00 : Intérêt des caroténoïdes dans le diagnostic et le suivi des mal-digestions. H. Faure et groupe de travail, Grenoble

10h30: Evaluation et choix des analyseurs de Cyto-hématologie : apport des descriptifs standardisés. A. Gruson et groupe de travail, Arras

$11 \mathrm{~h} 00$ : Evaluation analytique des réactifs de dosage des IgE spécifiques. JC. Renversez et groupe de travail « Allergie », Grenoble

11h30: Remise du prix SFBC

12h00: Assemblée générale de la SFBC

Modérateur : S. Lehmann (Montpellier)

Thème : L'enjeu protéomique

14h00 : De la protéomique fondamentale à la protéomique clinique, un long chemin semé d'améliorations technologiques et bioinformatiques. S. Lehman, Montpellier

14h45: Puces à protéines et ELISA multiple dans les laboratoires d'analyse biochimique. AM. Dupuy, Montpellier

15h30: Mise en place d'une plateforme de protéomique clinique ; exemple du Centre d'Innovation Biologique du CHU de Grenoble. M. Seve, Grenoble

16h15: De la phase pré-analytique à la phase analytique en protéomique clinique. K. Peoc'h, Paris

17h00 : Fin de la session

Journée du Secrétariat de Laboratoire

Jeudi 3 novembre

14h15: Introduction. F. Moreau, Paris

14h20: La nomenclature des actes de biologie médicale (NABM), ses pièges, les cumuls interdits, les examens que l'on peut rajouter à l'initiative du biologiste. Mme Kuhn ou Mme Robba, CNAMTS, Paris; JP. Clavel, SDB, Paris

15h15: Les conditions d'acceptation des prélèvements-Analyse des causes de non conformité A. Suiro, Bio-Qualité, Paris

16h15: Les causes de rejet lors des télétransmissions en IRIS B2 et en Sesam-Vitale. Mr Achard, CPAM, Versailles

Journées Biologiques de Lariboisière

Vendredi 4 novembre

Modérateur : P. Chappuis (Paris)

Session I : La médecine régénératrice : jusqu'où aller ?

09h00 : Présentation des Journées Biologiques de Lariboisière.

P. Chappuis, Paris

09h15: Conséquences pour la recherche de la révision, en 2004, des lois françaises de Bioéthique : clonage reproductif et thérapeutique, recherche sur cellules souches embryonnaires, extension du DPI, non brevetabilité des éléments du corps humain, situation internationale sur ces sujets. PL. Fagniez, Paris

09h55: Vers la production d'embryons humains obtenus par clonage : actualités et perspectives du clonage thérapeutique humain. W. S. Hwang, Séoul (Traduction simultanée)
Session II : La médecine régénératrice : jusqu'où aller ?

11h15: Les cellules souches : espoir thérapeutique et avenir. L. Coulombel, Créteil

Session III : Mycologie, bactéries multirésistantes, cliniques des traitements anticoagulants; frottis liquides et recherche du Papilloma virus dans le dépistage des cancers cervicaux

14h30: Cérémonie de remise du prix Henri Thouvenin 2005. M. Paris, Paris

15h00: La démarche diagnostique en mycologie.

D. Chabasse, Angers

15h35: Les bactéries multirésistantes : un danger. V. Jarlier, Paris

16h05: Les cliniques des traitements anticoagulants :

un réseau médecins-biologistes. L. Drouet, Paris

16h40: Place du frottis cervico-utérin par recueil des cellules en milieu liquide dans le dépistage du cancer du col de l'utérus. B. Cochand-Priollet, Paris

17h10: Place de la recherche des Papilloma virus par PCR dans le dépistage des cancers du col de l'utérus. C. Clavel, Reims

Groupe Formation SFBC

Vendredi 4 novembre

Thème : Difficultés en diagnostic sérologique et pour l'examen

cyto-bactériologique des urines

Modérateur : Y. Piémont (Strasbourg)

Session I : Limites et perspectives du diagnostic sérologique en Bactériologie à l'ère de l'amplification génique in vitro

09h45: Infections à Chlamydia trachomatis - Infections à Mycoplasma pneumoniae et à Chlamydia pneumoniae. B. de Barbeyrac, Bordeaux

10h15: Infections à Legionella - Infections à Bartonella henselae. B. Jaulhac, Strasbourg

10h45: Discussion

Session II : L'examen cyto-bactériologique des urines : une analyse simple ou bien complexe?

11h00: Méthodes bactériologiques accélérant le rendu de l'ECBU en pratique hospitalière et extra-hospitalière. R. Courcol, Lille

11h35: Interprétation de l'ECBU : critères, conditions pratiques d'application. P. Laudat, Tours

12h20: Conclusion

Journée ABTL / Techniciens

Vendredi 4 novembre

14h00: Introduction. J. Lazare, SDB, Metz

14h15: Du bilan martial de première intention aux diagnostics P. Brissot, CHU de Rennes, Rennes

15h00: Hémostase : Actualités, Bonnes pratiques, Perspectives

- Le prélèvement et son traitement

- La surveillance des traitements anticoagulants

- Le dépistage des anomalies à risque hémorragique

- Le bilan de thrombose

- Les nouveaux tests

M. Alhenc-Gelas, Hôpital Georges Pompidou, Paris

16h00: Point d'information :

- La réforme de l'Assurance Maladie. H. De Boisseu, Paris

- La licence professionnelle J. Potdevin, ESTBA, Paris

- Les prélèvements des techniciens à domicile J. Bégué, SDB, Paris

17h00: La responsabilité professionnelle des techniciens

C. Roquelle-Meyer, Paris, O. Saumon, Paris

Journée du Syndicat des Biologistes

Samedi 5 novembre

09h30 : L'apport de la biologie médicale dans la démarche économique actuelle. A. Fléchel, SFRL, Paris

10h30 : La réforme de l'Assurance Maladie : un an après J. Benoit, SDB, Paris

Renseignements et inscriptions

Conférences

Reed Expositions France

70 rue Rivay, 92532 Levallois-Perret cedex

Contact : Manon GOUSSET

Tél. : 0147562108 / Fax : 0147565258

manon.gousset@reedexpo.fr

Exposition

Le salon est ouvert du jeudi 3 au samedi 5 novembre 2005

Tous les jours, de 9 h00 à 19 h00, excepté le samedi de 9 h00 à 17 h00. 